05,13

\title{
Термоиндуцированное изменение поля переключения планарных CoNi-микрочастиц, сформированных на поверхности монокристаллического ниобата лития
}

\author{
() Д.А. Бизяев ${ }^{1}$, Н.И. Нургазизов ${ }^{1}$, А.А. Бухараев ${ }^{1}$, А.П. Чукланов ${ }^{1}$, В.Я. Шур ${ }^{2}$, А.Р. Ахматханов ${ }^{2}$ \\ ${ }^{1}$ Казанский физико-технический институт им. Е.К. Завойского Казанского научного центра РАН, \\ Казань, Россия \\ ${ }^{2}$ Институт естественных наук и математики УрФУ, \\ Екатеринбург, Россия \\ E-mail: dbiziaev@inbox.ru
}

Поступила в Редакцию 9 апреля 2021 г.

В окончательной редакции 9 апреля 2021 г.

Принята к публикации 19 апреля 2021 г.

Методами магнитно-силовой микроскопии были исследованы магнитные свойства (поле переключения) и переходы из многодоменного в однодоменное состояние планарных микрочастиц $\mathrm{Co}_{18} \mathrm{Ni}_{82}$ размером $7.5 \times 7.5 \times 0.03 \mu \mathrm{m}^{3}$ при различных температурах. Использование в качестве подложки гексагонального монокристалла ниобата лития, обладающего отличающимися температурными коэффициентами линейного расширения вдоль разных кристаллографических осей, позволило индуцировать одноосные механические напряжения в микрочастицах путем относительно небольшого нагрева или охлаждения образца по сравнению с его температурой напыления. Показано, что за счет термоиндуцированного магнитоупругого эффекта увеличение температуры всего на $50 \mathrm{~K}$ может привести к семикратному уменьшению величины поля переключения.

Ключевые слова: магнитоупругий эффект, магнитная силовая микроскопия, перемагничивание, ниобат лития, температура.

DOI: 10.21883/FTT.2021.09.51252.13H

\section{1. Введение}

В последнее время достаточно широкое применение для записи и хранения информации получили магнитные носители. В качестве бита информации у таких носителей используется направление намагниченности небольшой области сплошной магнитной пленки или отдельной микро- или наночастицы [1,2]. При этом к таким устройствам предъявляются требования противоположные по своей сути. С одной стороны, носитель должен быть как можно более устойчивым к внешним факторам для длительного хранения записанной информации, т.е. обладать как можно более высокой коэрцитивной силой. С другой стороны, при записи информации данный параметр должен быть как можно более низким для того, чтобы уменьшить количество расходуемой при этом энергии. Так как запись информации происходит обычно при помощи внешнего магнитного поля, то даже небольшое снижение коэрцитивной силы на это время может привести к существенному увеличению энергоэффективности устройства. Для такого снижения можно использовать разные подходы. Один из подходов предполагает локальный нагрев носителя, и получил название термоассистируемой магнитной записи информации (Heat Assisted Magnetic Recording) [3,4]. В качестве носителя может выступать паттернированная среда, состоящая из частиц. Кроме этого, для управления намагниченностью этих частиц можно использовать маг- нитоупругий эффект $[5,6]$. Создавая в частице одноосные механические напряжения, можно не только снизить ее коэрцитивную силу, но и изменять направление намагниченности без приложения внешнего поля, что позволяет существенно улучшить энергоэффективность таких устройств [7]. Данное направление исследований получило название стрейнтроника (от английского слова „strain“-напряжение). Обычно в стрейнтронных устройствах для создания механических напряжений в частицах используются подложки из пьезоэлектрических материалов, которые могут существенно изменять свои размеры в определенном направлении под действием электрического поля. В тоже время, у некоторых кристаллов (не обязательно пьезоэлектриков) наблюдается неравномерное термическое расширение по разным осям. Если создать на таких кристаллах магнитные частицы, то их коэрцитивную силу можно будет уменьшать локальным нагревом подложки и энергоэфективность такого подхода будет существенно выше, чем у HAMR-метода. В этом случае коэрцитивная сила будет меняться не только за счет снижения намагниченности насыщения материала, из которого изготовлена частица (как в случае с HAMR), но и за счет наводимой магнитоупругой анизотропии.

Недавно нами было показано что, используя в качестве подложки монокристалл ниобата лития $\left(\mathrm{LiNbO}_{3}\right)$ в гексагональной фазе, можно реализовать переход планарных CoNi-микрочастиц с латеральными размерами 
$25 \times 25 \mu \mathrm{m}^{2}$ из многодоменного в квазиоднородно намагниченное состояние. Это происходит за счет наведения в них магнитоупругой одноосной анизотропии, вызванной анизотропным термическим расширением подложки при нагреве [8].

В настоящей работе была исследована подобная система, представляющая собой планарные микрочастицы CoNi квадратной формы, которые были сформированы на поверхности гексагонального монокристалла $\mathrm{LiNbO}_{3}$. Методом магнитно-силовой микроскопии была определена величина поля переключения таких микрочастиц при температурах от комнатной и выше. Было показано, что небольшое изменение температуры монокристаллической подложки может приводить к многократному изменению величины поля переключения таких микрочастиц.

\section{2. Создание образцов и методика проведения исследований}

Для проведения исследований были выбраны микрочастицы $\mathrm{CoNi}$ квадратной формы размерами $7.5 \times 7.5 \times 0.03 \mu \mathrm{m}^{3}$. Ранее на подобных микрочастицах, сформированных на поверхности стеклянной подложки, были проведены исследования зависимости распределения локальной намагниченности от величины одноосного механического напряжения [5], что давало возможность проводить сравнение полученных результатов между собой. Изучение распределения намагниченности в микрочастицах и их топографии проводилось с помощью сканирующего зондового микроскопа (С3М) Solver P47 (NT MDT) в режимах атомно-силовой и магнитно-силовой микроскопии (АСМ и МСМ соответственно). Используемый СЗМ был дополнительно оборудован электромагнитом и термоячейкой, что позволяло в процессе МСМ-измерений создавать постоянное магнитное поле до $\pm 16 \mathrm{mT}$ и нагревать образец до 370 К. МСМ-измерения образца проводились по однопроходной методике (когда во время сканирования зонд находится на постоянном удалении (примерно $200 \mathrm{~nm}$ ) от исследуемой поверхности) для того, чтобы снизить возможное влияние МСМ-зонда на распределение намагниченности в микрочастицах. Для проведения исследований использовались стандартные коммерческие кантилеверы „Multi75M-G“ (BudgetSensors).

В настоящей работе для изучения магнитных свойств CoNi-микрочастиц использовалась подложка из монокристалла $\mathrm{LiNbO}_{3}$ в гексагональной фазе размером $9 \times 3 \times 1 \mathrm{~mm}^{3}$. Кристалл был изготовлен таким образом, что кристаллографическая ось ,, $c^{\text {“ }}$ и одна из осей ,, $a^{\text {“ }}$ лежали в одной плоскости и были взаимно перпендикулярны. На этой плоскости формировались микрочастицы. Согласно литературным данным температурный коэффициент линейного расширения $\left(\alpha_{1}\right)$ вдоль

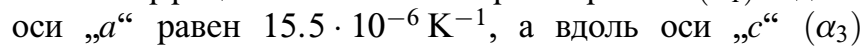
составляет $7.5 \cdot 10^{-6} \mathrm{~K}^{-1}[9,10]$, то есть при нагревании кристалл расширялся вдоль оси „, $a^{“}$ примерно в 2 раза больше. Магнитный сплав, из которого изготавливались микрочастицы, имел состав Со $18 \%$, Ni 82\%, константу магнитострикции насыщения $\lambda_{s}=-25 \cdot 10^{-6}[11]$ и изотропный температурный коэффициент линейного расширения для поликристаллической фазы равный $13 \cdot 10^{-6} \mathrm{~K}^{-1}[12]$.

Металл осаждался в вакууме $4 \cdot 10^{-6} \mathrm{~Pa}$ методом распыления твердотельной мишени электронным лучом как при комнатной температуре, так и на подогретую до $320 \mathrm{~K}$ подложку. Плотно прижатая к поверхности подложки металлическая сетка с квадратными отверстиями использовалась в качестве маски. Сетка была ориентирована таким образом, чтобы две противоположные стороны осажденной квадратной микрочастицы были параллельны оси „, $a^{\text {“ }}$ подложки. Поскольку длинная сторона подложки была параллельна оси „a“, образец легко ориентировался при исследованиях. Микрочастицы имели поликристаллическую структуру. Площадь заполнения поверхности подложки микрочастицами составляла $4 \mathrm{~mm}^{2}$, что позволило исследовать сразу ансамбль микрочастиц.

После осаждения микрочастиц вся поверхность образца покрывалась сплошной поликристаллической $\mathrm{CoNi}$ пленкой толщиной 3-5nm, которая была необходима для нейтрализации электростатического взаимодействия СЗМ зонда с образцом. При проведении измерений эта пленка и СЗМ-зонд заземлялись. Пленка создавалась из того же материала, что и микрочастицы для того, чтобы иметь одинаковые температурные коэффициенты линейного расширения. Использование других проводящих пленок для нейтрализации заряда кристалла могло привести к созданию дополнительных механических напряжений на микрочастицах при изменении температуры. Пленка $\mathrm{CoNi}$ имела анизотропию в виде „легкая плоскость“.

Предполагалось, что анизотропное сжатие подложки в результате охлаждения образца до комнатной температуры создает в микрочастицах и в пленке одноосное механическое напряжение растяжения, направленное вдоль оси „,с кристалла. Нагревание образца выше комнатной температуры приводит сначала к уменьшению наведенных напряжений, а при температурах свыше $320 \mathrm{~K}-$ к появлению одноосных механических напряжений сжатия вдоль оси „с“ кристалла. В работе представлены результаты изучения управления анизотропией, наведенной описанным выше способом, которая приводит к существенной перестройке структуры намагниченности микрочастицы.

\section{3. Экспериментальные результаты и их обсуждение}

На первом этапе исследований использовался образец, на котором микрочастицы $\mathrm{CoNi}$ формировались на поверхности $\mathrm{LiNbO}_{3}$ при комнатной температуре $(300 \mathrm{~K})$. Типичное АСМ-изображение микрочастиц, полученное СЗМ в полуконтактном режиме, приведено на 


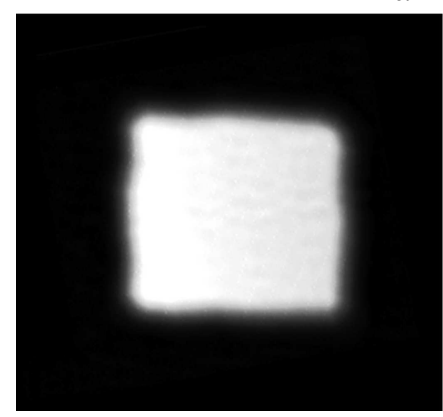

$b$

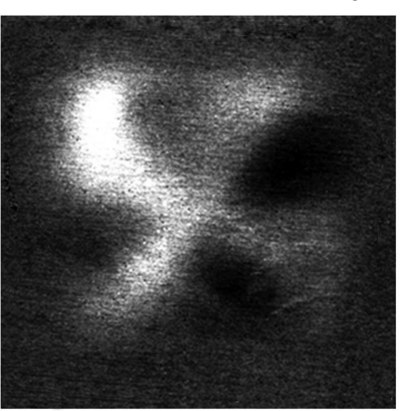

$c$

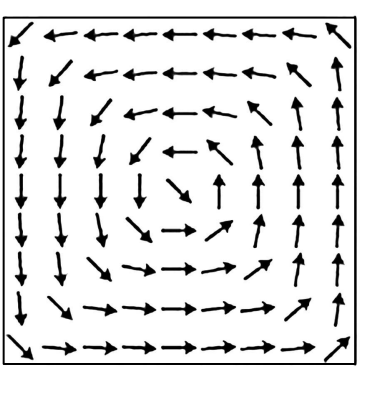

$d$

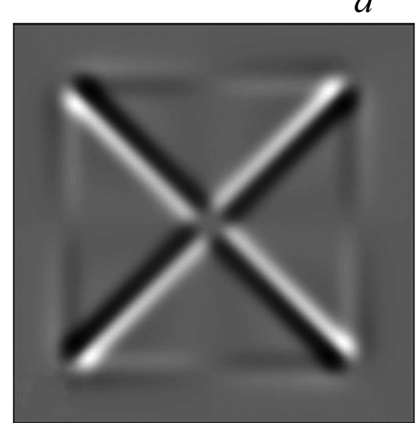

Рис. 1. АСМ изображение $7.5 \times 7.5 \times 0.03 \mu \mathrm{m}^{3}$ СоNi-микрочастицы $-(a)$ и ее МСМ-изображение - $(b)$ без действующих механических напряжений при комнатной температуре $(300 \mathrm{~K})$. Распределение намагниченности в такой микрочастице, полученное с помощью компьютерного моделирования в программе OOMMF $-(c)$, и виртуальное МСМ-изображение $-(d)$, построенное по этому распределению. Размер области сканирования $13 \times 13 \mu \mathrm{m}^{2}$.

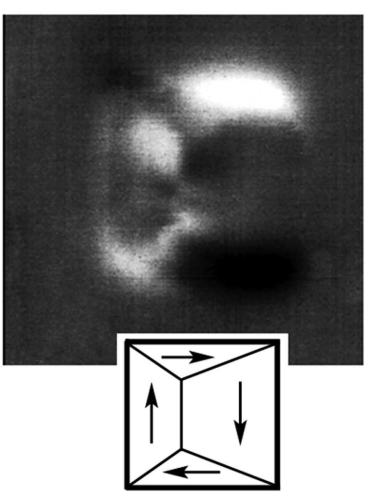

$e$

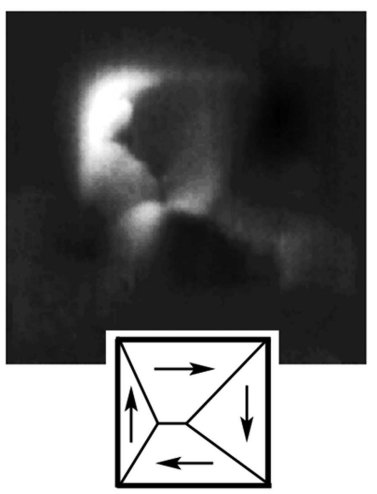

$b$

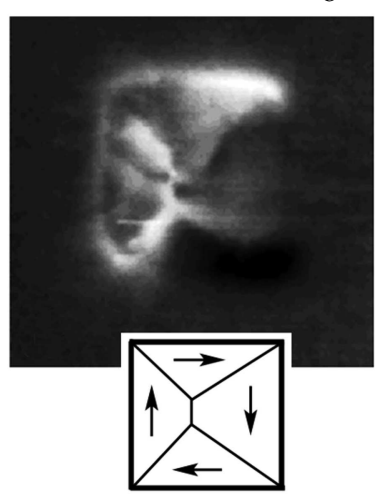

$f$

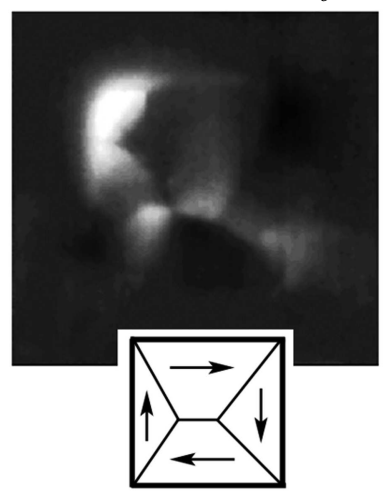

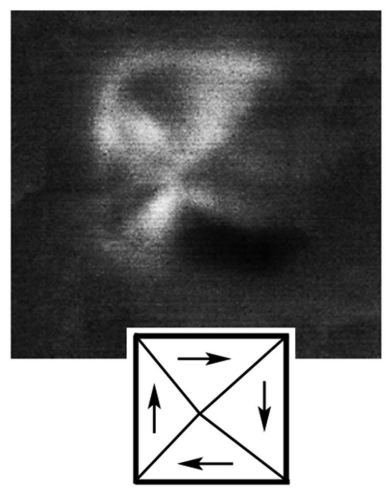

$g$

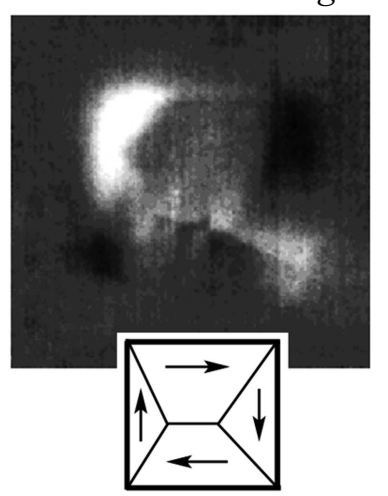

$d$

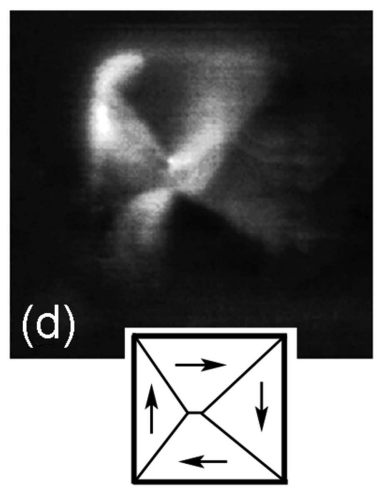

$h$

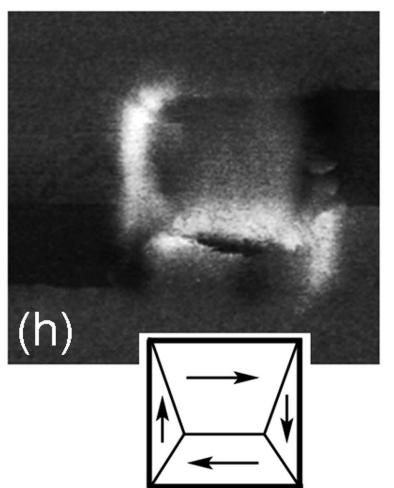

Рис. 2. МСМ-изображения $7.5 \times 7.5 \times 0.03 \mu \mathrm{m}^{3}$ СоNi-микрочастицы, полученные при температуре $300 \mathrm{~K}-(a)$, $310 \mathrm{~K}-(b)$, $320 \mathrm{~K}-(c), 330 \mathrm{~K}-(d), 340 \mathrm{~K}-(e), 350 \mathrm{~K}-(f), 360 \mathrm{~K}-(g)$ и $370 \mathrm{~K}-(h)$. Соответствующие схемы распределения намагниченности показаны во вставках под каждым рисунком. Размер области сканирования $13 \times 13 \mu \mathrm{m}^{2}$.

рис. 1, а. При комнатной температуре на микрочастицы не действовали механические напряжения, связанные с термическим расширением подложки. Типичное для такого случая МСМ-изображение микрочастицы приведено на рис. $1, b$. Как показало компьютерное моделирование распределения намагниченности (рис. 1,c) с помощью программного пакета OOMMF [13], оно соответствует классической четырех доменной структуре (так называемой структуре Ландау). Модельное МСМизображение (рис. $1, d$ ), построенное на основе данного распределения при помощи программы „Виртуальный микроскоп“ [14] хорошо совпадало с наблюдаемым экспериментально и подтвердило первоначальные выводы о магнитной структуре микрочастицы.

Ранее на подобных микрочастицах, сформированных на поверхности стеклянной подложки, были проведены 
исследования зависимости распределения локальной намагниченности от величины одноосного механического напряжения [5]. МСМ-изображения для микрочастиц на стеклянной подложке и на $\mathrm{LiNbO}_{3}$, (рис. 1, $b$ ), на которые не действуют одноосные механические напряжения, совпадали, что подтверждало отсутствие механических напряжений в микрочастицах.

На втором этапе использовался образец с микрочастицами, сформированными при температуре $320 \mathrm{~K}$. Для проведения исследований температурной зависимости распределения намагниченности CoNi-микрочастиц в зависимости от температуры образец нагревался в термоячейке. МСМ-измерения проводились без внешнего магнитного поля, от комнатной температуры с шагом $5 \mathrm{~K}$. Область сканирования включала в себя изображения четырех микрочастиц. При комнатной температуре $(300 \mathrm{~K})$ на МСМ-изображениях наблюдался увеличенный размер доменов, направление намагниченности которых совпадало с осью „A“ кристаллической подложки (рис. 2, $a$, для наглядности приводится изображение только одной микрочастицы). Между этими доменами наблюдалась характерная доменная стенка (перемычка). Так как материал микрочастиц имел отрицательную константу магнитострикции, можно сделать вывод о том, что микрочастицы находятся под действием одноосного растягивающего напряжения, направление которого совпадает с осью „с“ кристалла, т. е. увеличивается размер доменов, направление намагниченности в которых перпендикулярно направлению растяжения. При увеличении температуры размеры этих доменов сокращаются (рис. 2,b) и при температуре $315 \mathrm{~K}$ все домены становятся приблизительно одинаковыми, а характерная перемычка между ними исчезает (рис. 2, c). Это говорит о том, что одноосное механическое напряжение становится настолько мало, что перестает оказывать влияние на магнитную структуру микрочастицы. При температуре свыше $325 \mathrm{~K}$ на МСМ-изображениях наблюдается увеличение размера доменов направление намагниченности, которых совпадает с осью „с“ кристалла (рис. 2, $d$ ). Это позволяет сделать вывод о том, что происходит сжатие микрочастиц в данном направлении. Величина этого сжатия растет с повышением температуры (рис. 2,e-h). Между доменами также наблюдается характерная перемычка, направленная вдоль оси „с“ кристалла, которая увеличивается при росте температуры.

Полученные на этом этапе данные подтвердили первоначальное предположение о возможности регулирования одноосных механических напряжений, действующих на микрочастицы, при помощи изменения температуры монокристаллической подложки. Согласно полученным данным, наводимая одноосная анизотропия не влияет на магнитную структуру CoNi-микрочастиц в интервале температур 315-325 K, что позволило дополнительно проконтролировать температуру напыления микрочастиц и определить ее равной $320 \pm 5 \mathrm{~K}$.

На третьем этапе проведения исследований определялась величина поля переключения микрочастиц и ее изменение при разных температурах образца (шаг по температуре - $10 \mathrm{~K}$ ). Для этого образец с микрочастицами нагревался до определенной температуры. Затем включалось внешнее магнитное поле индукцией $+16 \mathrm{mT}$, направленное перпендикулярно оси „с“ кристалла, которое приводило к квазиоднородному намагничиванию микрочастиц в данном направлении. Далее происходило постепенное (с шагом $0.5 \mathrm{mT}$ ) уменьшение поля. На каждом шаге получалось МСМ-изображение. В качестве примера на рис. 3, $а$ приведено МСМ-изображение микрочастицы в таком квазиоднородном состоянии намагничивания, полученное при температуре $320 \mathrm{~K}$ в магнитном поле $+6 \mathrm{mT}$. В магнитных полях ниже определенной величины квазиоднородная намагниченность разрушалась, и микрочастица становилась многодоменной (рис. $3, b$ ). Уменьшение внешнего магнитного поля до нуля не приводило к появлению исходного распределения намагниченности в микрочастице, которое наблюдалось на первом этапе исследований (см. рис. $1, b$, $2, c)$, что обусловлено наличием гистерезиса. После достижения нулевого значения направление внешнего магнитного поля изменялось на противоположное и начиналось его последовательное увеличение с тем же шагом (рис. 3,c). При определенной индукции внешнего поля многодоменная структура микрочастицы исчезала. Осуществлялся переход в квазиоднородно намагниченное состояние (рис. 3,d). Данное значение индукции внешнего магнитного поля принималось за величину поля переключения микрочастицы $\left(B_{s w}\right)$. Дальнейшее увеличение магнитного поля практически не изменяло МСМ-изображения микрочастицы.

Следует отметить, что при больших механических напряжениях (при сильном отличии температуры исследования от температуры приготовления образца) микрочастицы оставались квазиоднородно намагниченными в полях до $+16 \mathrm{mT}$. При смене направления внешнего магнитного поля квазиоднородное состояние и направление намагниченности микрочастиц сохранялось до определенного значения индукции, при котором намагниченность частицы изменялась на противоположную без промежуточного многодоменного состояния (рис. $3, e-h)$.

Полученные величины $B_{s w}$ представлены в виде графика на рис. 4. Следует отметить, что величина $B_{s w}$ при температуре $320 \mathrm{~K}$ соответствует полю переключения микрочастиц в случае, когда на них не действует одноосное механическое напряжение. Это позволяет анализировать влияние механических напряжений на перемагничивание микрочастиц. При температуре ниже $320 \mathrm{~K}$ растяжение микрочастиц в направлении перпендикулярном направлению внешнего магнитного поля за счет отрицательной магнитострикции CoNi-сплава, приводит к увеличению $B_{s w}$ микрочастиц (рис. 4).

При температуре выше $320 \mathrm{~K}$ микрочастицы сжимаются в направлении, перпендикулярном магнитному полю, что приводит к уменьшению $B_{s w}$ микрочастиц. При нагреве образца от 300 до $350 \mathrm{~K}$ (всего на $50 \mathrm{~K}$ ) поле переключения микрочастиц уменьшается в семь 

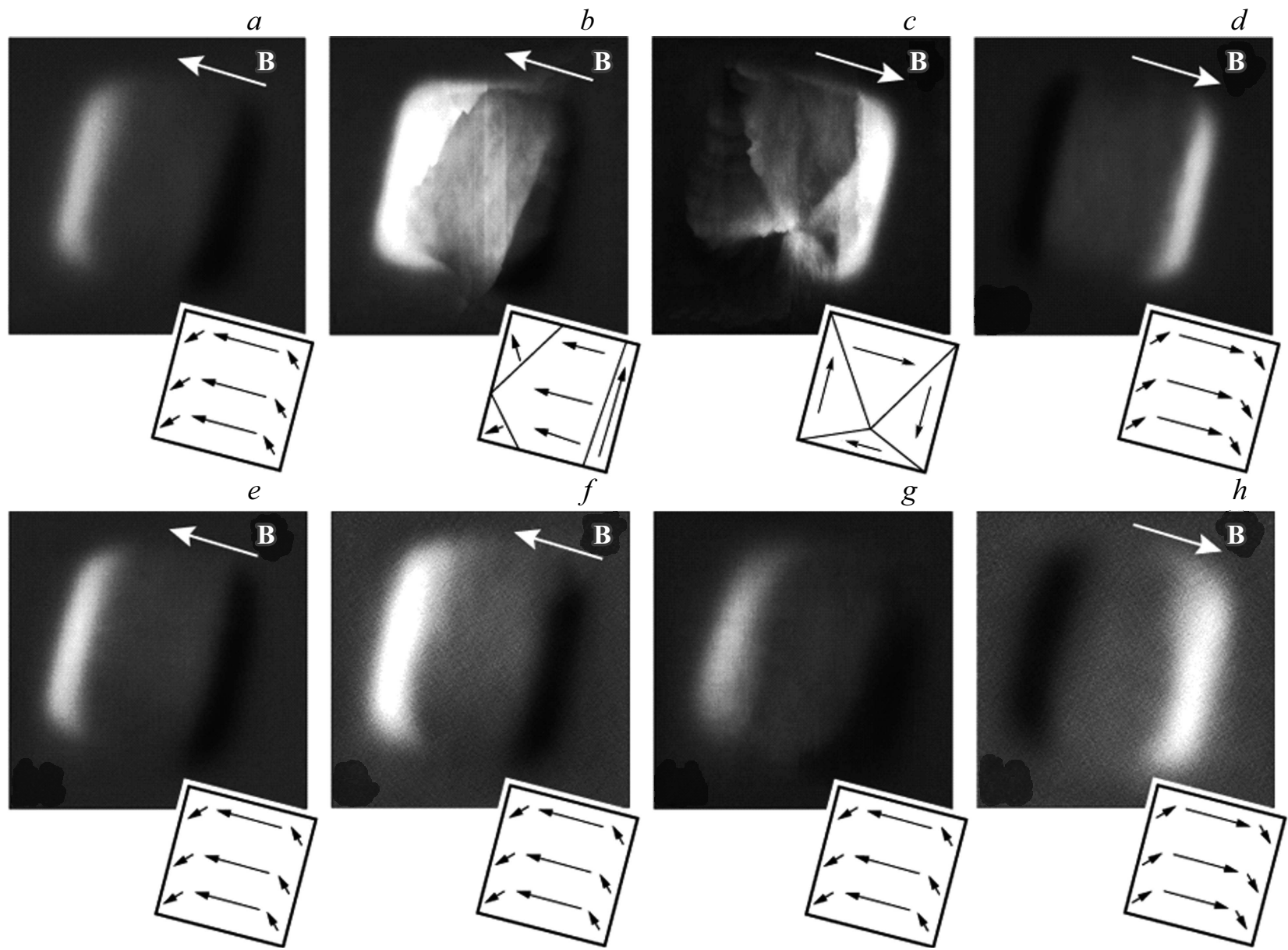

Рис. 3. МСM изображения $7.5 \times 7.5 \times 0.03 \mu \mathrm{m}^{3}$ CoNi-микрочастицы при температуре $320 \mathrm{~K}$ во внешнем магнитном поле индуктивностью $(a):+6 \mathrm{mT} ;(b):+2 \mathrm{mT} ;(c):-2 \mathrm{mT}$ и $(d):-7.5 \mathrm{mT}$. МСМ изображения микрочастицы при температуре $350 \mathrm{~K}$ во внешнем магнитном поле индуктивностью $(e):+6 \mathrm{mT} ;(f):+2 \mathrm{mT} ;(g): 0 \mathrm{mT}$ и $(h):-2 \mathrm{mT}$. Соответствующие схемы распределения намагниченности показаны во вставках под каждым рисунком. Белыми стрелками показано направление действия внешнего магнитного поля В. Размер области сканирования $13 \times 13 \mu \mathrm{m}^{2}$.

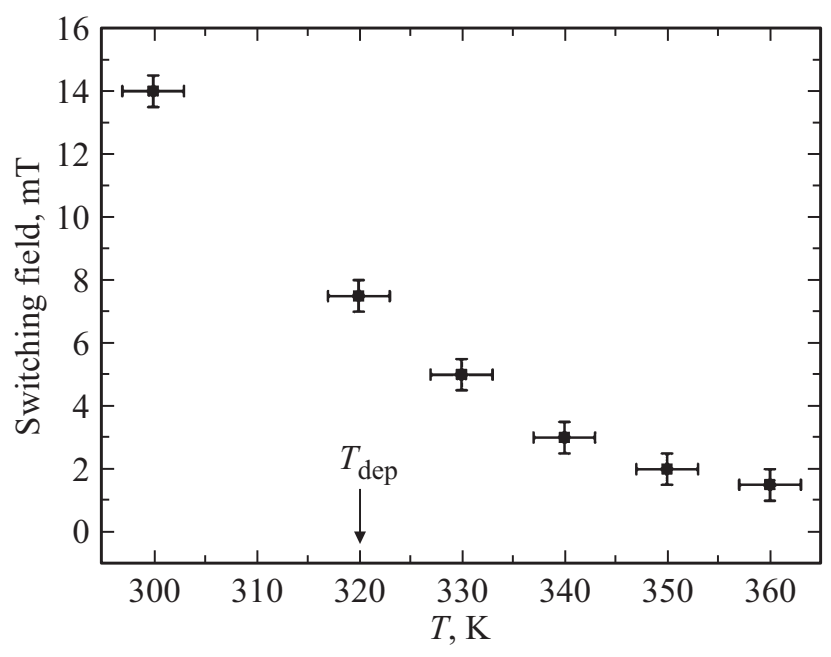

Рис. 4. Зависимость величины поля переключения $\left(B_{s w}\right)$ $7.5 \times 7.5 \times 0.03 \mu \mathrm{m}^{3}$ CoNi-микрочастицы от температуры образца. $T_{d e p}=320 \mathrm{~K}-$ температура напыления образца. раз. Таким образом, напыление микрочастиц на $\mathrm{LiNbO}_{3}$ подложку при температурах выше комнатной позволяет повысить величину поля переключения микрочастиц при комнатной температуре за счет наводимой магнитоупругой анизотропии. Нагрев образца выше температуры его напыления уменьшает поле переключения микрочастиц.

\section{4. Заключение}

Проведенные исследования показали, что предложенный метод управления полем переключения микрочастиц с помощью термоиндуцированного магнитоупругого эффекта может оказаться эффективным при использовании в устройствах термоассистируемой магнитной записи информации. На примере планарных $\mathrm{CoNi}$ микрочастиц, сформированных на поверхности $\mathrm{LiNbO}_{3}$, было показано, что за счет небольшого изменения температуры образца (с 320 до $350 \mathrm{~K}$ ) можно более чем в три раза уменьшить поле переключения микрочастиц (от 7.5 до $2 \mathrm{mT}$ ). Формирование микрочастиц при тем- 
пературах более высоких, чем температура их использования, также позволяет создавать в них магнитоупругую анизотропию, увеличивающую поле переключения микрочастиц. Представляется перспективным проведение дальнейших исследований термоиндуцированного магнитоупругого эффекта на однодоменных частицах с высоким значением константы магнитострикции.

\section{Финансирование работы}

Госзадание № АААА-А18-118041760011-2 — „Создание интеллектуальных систем и функциональных материалов для нано- и биотехнологий, элементной базы наноэлектроники и оптоэлектроники, устройств преобразования и хранения энергии. Диагностика дисперсных систем, наночастиц и материалов, включая наноматериалы“.

\section{Конфликт интересов}

Авторы заявляют, что у них нет конфликта интересов.

\section{Список литературы}

[1] K.M. Krishnan, T. Takeuchi, Y. Hirayama, D.M. Donnet, Y. Honda, M. Futamoto. J. Magn. Soc. Jpn. 18, S1_PMRC_94_1, S1_15 (1994).

[2] C.-Y. Liang, A. Sepulveda, S. Keller, G.P. Carman. J. Appl. Phys. 119, 11, 113903 (2016).

[3] M.H. Kryder, E.C. Gage, T.W. McDaniel, W.A. Challener, R.E. Rottmayer, G. Ju, Y.T. Hsia, M.F. Erden. Proc. IEEE 96, 11,1810 (2008).

[4] N.I. Nurgazizov, T.F. Khanipov, D.A. Bizyaev, A.A. Bukharaev, A.P. Chuklanov. Phys. Solid State 56, 9, 1817 (2014).

[5] Н.И. Нургазизов, Д.А. Бизяев, А.А. Бухараев, А.П. Чукланов. ФТТ 62, 9, 1503 (2020).

[6] A.P. Chuklanov, N.I. Nurgazizov, D.A. Bizyaev, T.F. Khanipov, A.A. Bukharaev, V.Yu. Petukhov, V.V. Chirkov, G.G. Gumarov. J. Phys. Conf. Ser. 714, 1, 012006 (1-4) (2016).

[7] A.K. Biswas, S. Bandyopadhyay, J. Atulasimha. Appl. Phys. Lett. 104, 23, 232403 (2014).

[8] D.A. Bizyaev, A.A. Bukharaev, N.I. Nurgazizov, A.P. Chuklanov, S.A. Migachev. Phys. Status Solidi Rapid Res. Lett. 2000256 (2020).

[9] Y.S. Kim, R.T. Smith. J. Appl. Phys. 40, 4637-4641 (1969).

[10] R.T. Smith, F.S. Welsh. J. Appl. Phys. 42, 2219 (1971).

[11] M. Yamamoto, S. Taniguchi. Sci. Rep. Res. Tohoku A 7, 35 (1955).

[12] G. Laplanche, P. Gadaud, C. Barsch, K. Demtroder, C. Reinhart, J. Schreuer, E.P. George. J. Alloys Compd. 746, 244 (2018).

[13] M.J. Donahue, D.G. Porter. OOMMF User's Guide, Version 1.0. Natl. Inst. Standards Technol., Gaithersburg, MD, USA( 1999). http://math.nist.gov/oommf.

[14] D.V. Ovchinnikov, A.A. Bukharaev. Tech. Phys. 46, 1014 (2001).

Редактор Д.В. Жуманов 